\title{
Beyond Positivity Bounds and the Fate of Massive Gravity
}

\author{
Brando Bellazzini, ${ }^{1,2}$ Francesco Riva, ${ }^{3,4}$ Javi Serra, ${ }^{4,5}$ and Francesco Sgarlata ${ }^{6}$ \\ ${ }^{1}$ Institut de Physique Théorique, Université Paris Saclay, CEA, CNRS, F-91191 Gif-sur-Yvette, France \\ ${ }^{2}$ Dipartimento di Fisica e Astronomia, Università di Padova, Via Marzolo 8, I-35131 Padova, Italy \\ ${ }^{3}$ Départment de Physique Théorique, Université de Genève, 24 quai Ernest-Ansermet, 1211 Genève 4, Switzerland \\ ${ }^{4}$ Theory Division, CERN, CH-1211 Geneva 23, Switzerland \\ ${ }^{5}$ Physik-Department, Technische Universität München, 85748 Garching, Germany \\ ${ }^{6}$ SISSA International School for Advanced Studies and INFN Trieste, via Bonomea 265 34136, Trieste, Italy
}

(Received 15 December 2017; published 17 April 2018)

\begin{abstract}
We constrain effective field theories by going beyond the familiar positivity bounds that follow from unitarity, analyticity, and crossing symmetry of the scattering amplitudes. As interesting examples, we discuss the implications of the bounds for the Galileon and ghost-free massive gravity. The combination of our theoretical bounds with the experimental constraints on the graviton mass implies that the latter is either ruled out or unable to describe gravitational phenomena, let alone to consistently implement the Vainshtein mechanism, down to the relevant scales of fifth-force experiments, where general relativity has been successfully tested. We also show that the Galileon theory must contain symmetry-breaking terms that are at most one-loop suppressed compared to the symmetry-preserving ones. We comment as well on other interesting applications of our bounds.
\end{abstract}

DOI: 10.1103/PhysRevLett.120.161101

The idea that physics at low energy can be described in terms of light degrees of freedom alone, which goes under the name of effective field theory (EFT), is one of the most satisfactory organizing principles in physics. The effect of ultraviolet (UV) dynamics is systematically accounted for in the resulting infrared (IR) EFT by integrating out heavy degrees of freedom, which generate an effective Lagrangian made of infinitely many local operators. Since the symmetries of the underlying UV theory are retained in the IR, EFTs are predictive even when the UV dynamics is unknown: only a finite number of symmetric operators contribute, at a given accuracy, to observable quantities.

Remarkably, extra information about the UV can always be extracted if the underlying Lorentz invariant microscopic theory is unitary, causal, and local. These principles are encoded in the fundamental properties of the $S$ matrix such as unitarity, analyticity, crossing symmetry, and polynomial boundedness [1,2]. These imply a UV-IR connection in the form of dispersion relations that link the (forward) amplitudes in the deep IR with the discontinuity across the branch cuts integrated all the way to infinite energy $[3,4]$. Unitarity ensures the positivity of such discontinuities, and in turn the positivity of (certain) Wilson coefficients associated with the operators in the

Published by the American Physical Society under the terms of the Creative Commons Attribution 4.0 International license. Further distribution of this work must maintain attribution to the author(s) and the published article's title, journal citation, and DOI. Funded by SCOAP ${ }^{3}$.
IR effective Lagrangian. This UV-IR connection can be used to show that coefficients with the "wrong" sign cannot be generated by a Lorentz invariant, unitary, causal, and local UV completion [5]: the corresponding EFT, even if compatible with the symmetries of the system, is thrown to the "swampland." Positivity bounds have found several applications, including the proof of the $a$ theorem [6,7]; the study of chiral perturbation theory [8] and $W W$ scattering; and theories of composite Higgs [9-14], quantum gravity [15], massive gravity [16-18], Galileons [18-21], inflation $[22,23]$, the weak gravity conjecture [24,25], and conformal field theory [26-28]. The approach has been recently extended to particles of arbitrary spin [18], leading to a general no-go theorem on the leading energy-scaling behavior of the IR amplitudes, with applications to massive gravity [16] and Goldstini [29-31]. References [21,32,33] extended this technique beyond the forward limit.

In this Letter, we show that qualitatively new bounds, stronger than standard positivity constraints, can be derived by taking into account the irreducible IR cross sections under the dispersive integral, which are calculable within the EFT. We discuss for what models our bounds can be important and focus explicitly on two relevant applications: the EFT for a weakly broken Galileon [34,35], and the ghost-free massive gravity theory [36,37]. Figure 1 gives a preview of our results for the latter.

Let us consider the center-of-mass 2-to-2 scattering amplitude $\mathcal{M}^{z_{1} z_{2} z_{3} z_{4}}(s, t)$, where the polarization functions are labeled $z_{i}$. The Mandelstam variables are defined by $s=-\left(k_{1}+k_{2}\right)^{2}, t=-\left(k_{1}+k_{3}\right)^{2}, u=-\left(k_{1}+k_{4}\right)^{2}$ and 


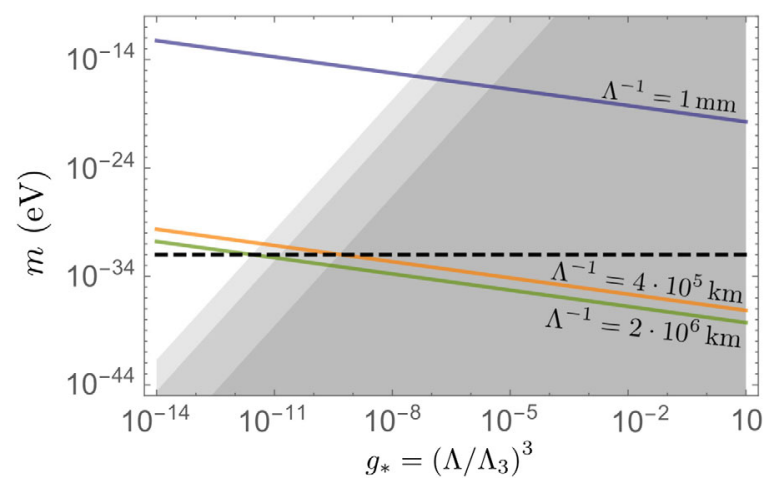

FIG. 1. Exclusion region for massive gravity in the plane of graviton mass $m$ and coupling $g_{*} \equiv\left(\Lambda / \Lambda_{3}\right)^{3}$, with $\Lambda$ being the physical cutoff and $\Lambda_{3}=\left(m^{2} m_{\mathrm{Pl}}\right)^{1 / 3}$ the strong coupling scale. Our theoretical bound Eq. (14) excludes with accuracy $\delta=1 \%$ the darkest gray region, and with $10 \%, 30 \%$ the gradually lighter gray regions. Solid lines mark the fixed cutoff $\Lambda$, whereas the dashed black line shows the upper experimental bound $m=10^{-32} \mathrm{eV}$. Our constraint gives rise to a tension between high $\Lambda$ and a small $m$ : the graviton mass can only be below the experimental bound at the expense of a premature breakdown of the EFT at macroscopically large distances.

satisfy $s+t+u=4 m^{2}$, where $m$ is the mass of the scattered particles. Our arguments will require finite $m \neq 0$, yet they hold even for some massless theories [scalars, spin- $1 / 2$ fermions, and softly broken $U(1)$ gauge theories], which have a smooth limit $m \rightarrow 0$. We call

$$
\mathcal{M}^{z_{1} z_{2}}(s) \equiv \mathcal{M}^{z_{1} z_{2} z_{1} z_{2}}(s, t=0)
$$

the forward elastic amplitude at $t=0$ and study the analyticity properties of $\mathcal{M}^{z_{1} z_{1}}(s) /\left(s-\mu^{2}\right)^{3}$, integrating along a closed contour $\Gamma$ in the complex $s$ plane, enclosing all the physical IR poles $s_{i}$ associated with stable light degrees of freedom entering the scattering (or its crossed symmetric process), together with the point $s=\mu^{2}$ lying on the real axis between $s=0$ and $s=4 m^{2}$. We define

$\Sigma_{\mathrm{IR}}^{z_{1} z_{2}} \equiv \frac{1}{2 \pi i} \oint_{\Gamma} d s \frac{\mathcal{M}^{z_{1} z_{2}}(s)}{\left(s-\mu^{2}\right)^{3}}=\sum \operatorname{Res}_{s=s_{i}, \mu^{2}}\left(\frac{\mathcal{M}^{z_{1} z_{2}}(s)}{\left(s-\mu^{2}\right)^{3}}\right)$,

which is calculable within the EFT. Using Cauchy's integral theorem, the contour can be deformed into a new contour that runs around the $s$-channel and $u$-channel branch cuts, and goes along a big circle eventually sent to infinity. The boundary contribution at infinity vanishes, due to the Froissart-Martin asymptotic bound $|\mathcal{M}(s \rightarrow \infty)|<$ const $\times s \log ^{2} s$, which is always satisfied in any local massive QFT $[38,39]$. This leads to a dispersion relation that connects the IR, Eq. (2), to an integral (UV) of the total cross section

$$
\begin{aligned}
\Sigma_{\mathrm{IR}}^{z_{1} z_{2}}= & \sum_{X} \int_{4 m^{2}}^{\infty} \frac{d s}{\pi} \sqrt{1-4 \frac{m^{2}}{s}} \\
& \times\left[\frac{s \sigma^{z_{1} z_{2} \rightarrow X}(s)}{\left(s-\mu^{2}\right)^{3}}+\frac{s \sigma^{-\bar{z}_{1} z_{2} \rightarrow X}(s)}{\left(s-4 m^{2}+\mu^{2}\right)^{3}}\right]_{\mathrm{IR}},
\end{aligned}
$$

where we use crossing symmetry relating the $s$ and $u$ channels in the forward limit even for particles with spin [18]: in the helicity basis, $\mathcal{M}^{z_{1} z_{2}}(s)=\mathcal{M}^{-\bar{z}_{1} z_{2}}\left(u=-s+4 m^{2}\right)$, and for particles that are their own antiparticles, $\bar{z}=z$. We also invoke the optical theorem to relate the imaginary parts of the amplitudes (across the branch cuts) to the cross section.

For any theory where particles 1 and 2 are interacting, as long as $0<\mu^{2}<4 m^{2}$, the right-hand side (rhs) of Eq. (3) is always positive, and one obtains the rigorous positivity bound, $\Sigma_{\mathrm{IR}}^{z_{1} z_{2}}>0$. Since $\Sigma_{\mathrm{IR}}^{z_{1} z_{2}}$ is calculable in the IR in terms of the Wilson coefficients, this provides a nontrivial constraint on the EFT.

We can in fact extract more than positivity bounds by noticing that the total cross section on the rhs of the dispersion relation Eq. (3) contains an irreducible contribution from IR physics, which is also calculable within the EFT, by construction. The other contributions, e.g., those from the UV, are incalculable with the EFT but are nevertheless always strictly positive, by unitarity. Moreover, each final-state $X$ in the total cross section contributes positively too. Therefore, an exact inequality follows from truncating the rhs of Eq. (3) at some energy $s_{\max } \equiv E^{2} \ll \Lambda^{2}$ below the cutoff $\Lambda$ of the EFT. To leading order (LO) in powers of $(E / \Lambda)^{2}$ and $(m / E)^{2}$ [hence also $\left.(\mu / E)^{2}\right]$, the bound in Eq. (3) becomes

$$
\begin{aligned}
\Sigma_{\mathrm{IR}, \mathrm{LO}}^{z_{\mathrm{I}} z_{2}}> & \sum_{X} \int^{E^{2}} \frac{d s}{\pi s^{2}}\left[\sigma^{z_{1} z_{2} \rightarrow X}(s)+\sigma^{z_{1}-\bar{z}_{2} \rightarrow X}(s)\right]_{\mathrm{IR}, \mathrm{LO}} \\
& \times\left[1+O\left(\frac{m}{E}\right)^{2}+O\left(\frac{E}{\Lambda}\right)^{2}\right],
\end{aligned}
$$

where the subscript IR highlights the fact that both sides of the equation are computable within the EFT; the main source of error for small masses is the truncation of the tower of higher-dimensional operators. Choosing $E$ at or slightly below the cutoff $\Lambda$ gives just an order-of-magnitude estimate for the bound $[18,21]$, as originally suggested in Ref. [19]. A rigorous bound can instead be obtained by choosing a sufficiently small $(E / \Lambda)^{2}$ : percent accuracy can be achieved already with $E / \Lambda \approx 1 / 10$.

The $\Sigma_{\mathrm{IR}}^{z_{1} z_{2}}$ must therefore be not only positive but strictly larger than something which is itself positive and calculable within the EFT. Moreover, we can include any final states $X$, elastic or inelastic: the more channels and information are retained, the more refined the bound will be. Notice that the 2-to-2 cross section retained on the rhs is obtained by integrating over $t$; thus, effectively, our bounds capture as well the behavior of the amplitude away from the forward limit. 
The implications of our bound Eq. (4) are particularly interesting in theories where the elastic forward amplitude $\mathcal{M}^{z_{1} z_{2}}$, appearing on the left-hand side (lhs), is parametrically suppressed compared to the nonforward or inelastic ones appearing on the rhs. The Galileon, massive gravity, the dilaton, and WZW-like theories, as well as other models where $2 \rightarrow 2$ is suppressed while $2 \rightarrow 3$ is not, are other simple examples of theories that get nontrivial constraints, on the couplings and/or masses of the corresponding EFTs, that include and go beyond the positivity of $\Sigma_{\mathrm{IR}}$. Even in situations without parametric suppression, our bound carries important information: it links elastic and inelastic cross sections that might depend on coefficients of the EFT.

Galileon.-The Lagrangian for the weakly broken Galileon [34,35],

$$
\begin{aligned}
\mathcal{L}=- & \frac{1}{2}\left(\partial_{\mu} \pi\right)^{2}\left[1+\frac{c_{3}}{\Lambda^{3}} \square \pi+\frac{c_{4}}{\Lambda^{6}}\left((\square \pi)^{2}-\left(\partial_{\mu} \partial_{\nu} \pi\right)^{2}\right)\right. \\
& \left.+c_{5}(\ldots)\right]+\frac{\lambda}{4 \Lambda^{4}}\left[(\partial \pi)^{2}\right]^{2}-\frac{m^{2}}{2} \pi^{2},
\end{aligned}
$$

with physical cutoff $\Lambda$, has suppressed symmetry-breaking terms $\lambda \ll c_{3}^{2}, c_{4}$ and $m^{2} \ll \Lambda^{2}$. Forward $2 \rightarrow 2$ scattering is controlled at $O\left(s^{2}\right)$ by the symmetry-breaking interactions: the lhs of Eq. (4) is $\Sigma_{\mathrm{IR}}=\left(\lambda / \Lambda^{4}\right)+\left(c_{3}^{2} m^{2} / 2 \Lambda^{6}\right)$. On the other hand, the hard scattering is controlled by the symmetry-preserving interactions, $\sigma^{\pi \pi \rightarrow \pi \pi} \approx$ $3\left(c_{3}^{2}-2 c_{4}\right)^{2} s^{5} /\left(5120 \pi \Lambda^{12}\right)$.

In the massless limit, or more generally for $c_{3}^{2} m^{2} / \Lambda^{2} \ll \lambda$ (a natural hierarchy, given that $\lambda$ preserves a shift symmetry while $m^{2}$ does not), the bound Eq. (4) shows not only that $\lambda$ must be positive, but (parametrically) at most one loop factor away from $\left(c_{3}^{2}-2 c_{4}\right) / 4$ :

$$
\lambda>\frac{3}{640} \frac{\left(c_{3}^{2}-2 c_{4}\right)^{2}}{16 \pi^{2}}\left(\frac{E}{\Lambda}\right)^{8} \text { for } \quad \frac{c_{3}^{2} m^{2}}{\Lambda^{2}} \ll \lambda .
$$

For a massive Galileon with negligible $\lambda$ and $c_{3} \neq 0$, one gets a lower bound on the mass,

$m^{2}>\Lambda^{2} \frac{3\left(c_{3}-2 c_{4} / c_{3}\right)^{2}}{320 \times 16 \pi^{2}}\left(\frac{E}{\Lambda}\right)^{8}$ for $\quad \frac{c_{3}^{2} m^{2}}{\Lambda^{2}} \gg \lambda$,

where $(E / \Lambda)^{8} \approx 10^{-2}$ for a $30 \%$ accuracy. Therefore, the Galileon symmetry-breaking terms cannot be arbitrarily suppressed, the general lesson being that $O\left(s^{2}\right)$ terms in the amplitude cannot be too suppressed compared to the $O\left(s^{3}\right)$ terms. The results of Eqs. (6) and (7) hold when loop effects are included, as they simply generate terms that are subleading, e.g., $\sim(m / \Lambda)^{6} c_{3}^{4} m^{2} / 16 \pi^{2} \Lambda^{6}$ in $\Sigma_{\mathrm{IR}}$ (recall that $m \ll E \ll \Lambda$ ).

Massive gravity.-The action for ghost-free massive gravity, also known as $\Lambda_{3}$ or dRGT massive gravity, is [36,37] (for reviews, see Refs. [40,41])

$$
S=\int d^{4} x \sqrt{-g}\left[\frac{m_{\mathrm{Pl}}^{2}}{2} R-\frac{m_{\mathrm{Pl}}^{2} m^{2}}{8} V(g, h)\right],
$$

where $m_{\mathrm{Pl}}=(8 \pi G)^{-1 / 2}$ is the reduced Planck mass, $g_{\mu \nu}=$ $\eta_{\mu \nu}+h_{\mu \nu}$ is an effective metric written in terms of the Minkowski metric $\eta_{\mu \nu}$ (with mostly + signature) and a spin-2 graviton field $h_{\mu \nu}$ in the unitary gauge, $R$ is the Ricci scalar for $g_{\mu \nu}$, and

$$
\begin{aligned}
V(g, h)= & b_{1}\left\langle h^{2}\right\rangle+b_{2}\langle h\rangle^{2}+c_{1}\left\langle h^{3}\right\rangle+c_{2}\left\langle h^{2}\right\rangle\langle h\rangle+c_{3}\langle h\rangle^{3} \\
& +d_{1}\left\langle h^{4}\right\rangle+d_{2}\left\langle h^{3}\right\rangle\langle h\rangle+d_{3}\left\langle h^{2}\right\rangle^{2}+d_{4}\left\langle h^{2}\right\rangle\langle h\rangle^{2} \\
& +d_{5}\langle h\rangle^{4}
\end{aligned}
$$

is the soft graviton potential, with $\langle h\rangle \equiv h_{\mu \nu} g^{\mu \nu},\left\langle h^{2}\right\rangle \equiv$ $g^{\mu \nu} h_{\nu \rho} g^{\rho \sigma} h_{\sigma \mu}$, etc. Absence of ghosts implies that the coefficients of this potential depend on just two parameters, $c_{3}$ and $d_{5}$; see Ref. [37,42].

Note that, though tempting, the results obtained above for the Galileon cannot be directly interpreted in the context of massive gravity (even if the Galileon is the longitudinal component of the massive graviton), since the IR dynamics is different: for example, in the scattering of the Galileon scalar mode, the helicity- 2 mode exchanged in the $t$ channel contributes as much as the scalar mode.

Since the graviton is its own antiparticle, it is convenient to express Eq. (4) in terms of linear polarizations $[15,16,18]$ :

$$
\Sigma_{\mathrm{IR}, \mathrm{LO}}^{z_{1} z_{2}}>\sum_{X} \frac{2}{\pi} \int^{E^{2}} \frac{d s}{s^{2}}\left[\sigma^{z_{1} z_{2} \rightarrow X}(s)\right]_{\mathrm{IR}, \mathrm{LO}}
$$

We adopt the basis of polarizations of Ref. [16] to calculate the amplitudes for different initial- and final-state configurations, finding that, generally, $\Sigma_{\mathrm{IR}}^{z_{1} z_{2}} \sim m^{2} / \Lambda_{3}^{6}$ is suppressed by the small graviton mass, with $\Lambda_{3} \equiv\left(m^{2} m_{\mathrm{Pl}}\right)^{1 / 3}$ the strong coupling scale [43]. For instance,

$$
\begin{aligned}
& \Sigma_{\mathrm{IR}}^{S S}=\frac{2 m^{2}}{9 \Lambda_{3}^{6}}\left(7-6 c_{3}\left(1+3 c_{3}\right)+48 d_{5}\right)>0, \\
& \Sigma_{\mathrm{IR}}^{V V}=\frac{m^{2}}{16 \Lambda_{3}^{6}}\left(5+72 c_{3}-240 c_{3}^{2}\right)>0, \\
& \Sigma_{\mathrm{IR}}^{V S}=\frac{m^{2}}{48 \Lambda_{3}^{6}}\left(91-312 c_{3}+432 c_{3}^{2}+384 d_{5}\right)>0 .
\end{aligned}
$$

In contrast, the hard-scattering limits of the amplitudes that enter the rhs of Eq. (9) are unsuppressed. For $s, t \gg m^{2}$, e.g., elastic amplitudes read

$$
\begin{aligned}
\mathcal{M}^{S S} & =\frac{s t(s+t)}{6 \Lambda_{3}^{6}}\left(1-4 c_{3}\left(1-9 c_{3}\right)+64 d_{5}\right), \\
\mathcal{M}^{V V} & =\frac{9 s t(s+t)}{32 \Lambda_{3}^{6}}\left(1-4 c_{3}\right)^{2}, \\
\mathcal{M}^{V S} & =\frac{3 t}{4 \Lambda_{3}^{6}}\left(c_{3}\left(1-2 c_{3}\right)\left(s^{2}+s t-t^{2}\right)-\frac{5 s^{2}+5 s t-9 t^{2}}{72}\right) .
\end{aligned}
$$


At this point, we choose the energy scale $E$ in Eq. (9) below the cutoff, $E \ll \Lambda$, so that the EFT calculation of the cross sections is trustworthy, and above the mass, $E \gg m$, so that the amplitudes Eq. (11) dominate such cross sections. We define $\delta \equiv(E / \Lambda)^{2}$, that controls the accuracy of the EFT calculation, and obtain

$$
F_{z_{1} z_{2}}\left(c_{3}, d_{5}\right)>\left(\frac{4 \pi m_{\mathrm{Pl}}}{m}\right)\left(\frac{g_{*}}{4 \pi}\right)^{4} \delta^{6},
$$

where $g_{*} \equiv\left(\Lambda / \Lambda_{3}\right)^{3}$ and, e.g.,

$$
\begin{aligned}
F_{S S} & =\left[960 \frac{7-6 c_{3}\left(1+3 c_{3}\right)+48 d_{5}}{\left(1-4 c_{3}\left(1-9 c_{3}\right)+64 d_{5}\right)^{2}}\right]^{3 / 2}, \\
F_{V V} & =\left[\left(\frac{2560}{27}\right) \frac{5+72 c_{3}-240 c_{3}^{2}}{\left(1-4 c_{3}\right)^{4}}\right]^{3 / 2}, \\
F_{V S} & =\left[\frac{80640\left(91-312 c_{3}+432 c_{3}^{2}+384 d_{5}\right)}{1975-29808 c_{3}\left(1-2 c_{3}\right)\left(1-4 c_{3}+8 c_{3}^{2}\right)}\right]^{3 / 2} .
\end{aligned}
$$

A more complete set of $\Sigma_{\mathrm{IR}}^{z_{1} z_{2}}, \mathcal{M}^{z_{1} z_{2}}$, as well as the resulting inequalities involving the $F_{z_{1} z_{2}}\left(c_{3}, d_{5}\right)$ functions, are reported in the Supplemental Material [44].

The inequalities following from Eq. (12) are the main result of this discussion and can be read in several ways: as constraints on the plane of the graviton potential parameters $\left(c_{3}, d_{5}\right)$ for a given graviton mass $m$ and ratio $\left(\Lambda / \Lambda_{3}\right)^{3} \equiv g_{*}$, as a constraint on $g_{*}$ for fixed $m$ at a given point in the $\left(c_{3}, d_{5}\right)$ region allowed by positivity, or equivalently as a bound on the graviton mass for fixed coupling at that point.

An important aspect of Eq. (12) is that it is possible to find an absolute maximum value of $g_{*}$ above which our bounds do not allow for a solution: we write Eq. (12) as $m>m_{\min } \propto 1 / F_{z_{1} z_{2}}\left(c_{3}, d_{5}\right)$ and note that at each point $\left(c_{3}, d_{5}\right)$ the bound is determined by the smallest $F_{z_{1} z_{2}}$. Now, the positivity constraints Eq. (10) provide a compact allowed region in the $\left(c_{3}, d_{5}\right)$ plane, within which the (continuous) function $\min \left\{F_{z_{1} z_{2}}\right\}\left(c_{3}, d_{5}\right)$ has a maximum. This corresponds to $\left(\hat{c}_{3}, \hat{d}_{5}\right) \approx(0.18,-0.017)$ and $F_{V S} \approx$ $4.6 \times 10^{6}$; thus, the most conservative bound

$$
m>10^{-32} \mathrm{eV}\left(\frac{g_{*}}{4.5 \times 10^{-10}}\right)^{4}\left(\frac{\delta}{1 \%}\right)^{6} .
$$

Taking $m=10^{-32} \mathrm{eV}$ as a benchmark experimental upper bound on the graviton mass (see Ref. [52] for a critical discussion), any value $g_{*} \gtrsim 4.5 \times 10^{-10}$ is excluded, irrespectively of the values of $\left(c_{3}, d_{5}\right)$, a situation that we summarize in Fig. 1. Slightly stronger bounds can be obtained by working with the nonelastic channels, while if we were to admit a slightly larger uncertainty, e.g., $\delta=5 \%$, the upper bound on $g_{*}$ would increase by 1 order of magnitude. Smaller values of the graviton mass, e.g., $m \simeq H_{0} \simeq 10^{-33} \mathrm{eV}$, as is customary in cosmology, require an even smaller coupling. For a given mass, only as $g_{*}$ is lowered sufficiently according to Eq. (14), a region allowed by our bounds eventually materializes inside the positivity region.

At this point, the crucial question is what the physical meaning of $g_{*}$ is, and if it can be arbitrarily small, $g_{*} \lesssim 10^{-10}$ [18]. To our knowledge, most literature of massive gravity has so far taken $\Lambda=\Lambda_{3}$, or $\Lambda \gg \Lambda_{3}$, corresponding to $g_{*} \gtrsim 1$. These values are now grossly excluded by our bounds. From a theoretical point of view, $\Lambda$ and $\Lambda_{3}$ scale differently with $\hbar$, so that their ratio actually changes when units are changed, in such a way that $g_{*}$ indeed scales like a coupling constant. This is analogous to the difference between a vacuum expectation value $v$ and the mass of a particle $\sim$ coupling $\times v$ (e.g., the $W$-boson mass $\left.m_{W} \sim g v\right)$. The point, then, is that the cutoff $\Lambda$ is a physical scale, which differs from $\Lambda_{3}$ that instead does not have the right dimension to represent a cutoff. Since $\Lambda_{3}^{-1} \approx 320 \mathrm{~km}\left(m / 10^{-32} \mathrm{eV}\right)^{-2 / 3}$, a very small coupling $g_{*}$ translates into a very low cutoff (large in units of distance),

$\Lambda \simeq\left(4.1 \times 10^{5} \mathrm{~km}\right)^{-1}\left(\frac{g_{*}}{4.5 \times 10^{-10}}\right)^{1 / 3}\left(\frac{m}{10^{-32} \mathrm{eV}}\right)^{2 / 3}$.

This is clearly problematic. For example, let us consider the experimental tests of massive gravity in the form of bounds on fifth forces from the precise measurements of the Earth-Moon precession $\delta \phi[41,53,54]$. Due to the Vainshtein screening $[55,56]$, which is generically dominated by the Galileon cubic interactions in the $\left(c_{3}, d_{5}\right)$ region allowed by our bounds, the force mediated by the scalar mode compared to the standard gravitational one is $F_{S} / F_{\mathrm{GR}} \sim\left(r / r_{V}\right)^{3 / 2}$, where $r_{V}=\left(M / 4 \pi m_{\mathrm{Pl}}\right)^{1 / 3} \Lambda_{3}^{-1}=$ $\left(M / 4 \pi m^{2} m_{\mathrm{Pl}}^{2}\right)^{1 / 3}$ is the Vainshtein radius associated with the (static and spherically symmetric) source under consideration, in this case the Earth, $M=M_{\oplus}$. Before our bound, one would find that at lunar distances, $r=r_{\oplus L} \approx 3.8 \times 10^{5} \mathrm{~km}$, the ratio of forces, and thus also the precession $\delta \phi \sim \pi\left(F_{S} / F_{\mathrm{GR}}\right)$, even if very small for $m=10^{-32} \mathrm{eV}$, would be borderline compatible with the very high accuracy of present measurements $\sim 10^{-11}$. Now our result in Eq. (15) shows that the EFT is not valid already for $r \sim 1 / \Lambda>r_{\oplus L}$. This implies that the Vainshtein screening should receive important corrections before reaching the (inverse) cutoff $1 / \Lambda$, and moreover, it means that new degrees of freedom should become active at that scale: two effects that likely impair the fifth-force suppression and hinder the agreement with the precise measurement of the Earth-Moon precession. (Note that the Vainshtein redressing [57] deep inside the Vainshtein region, $\Lambda_{3} \rightarrow z \Lambda_{3}$ with $z \gg 1$, does not generically extend to the physical cutoff $\Lambda$, since the rescaling of the kinetic term in a background does not affect the extra derivative 
terms that come without extra field insertions. In other words, a potentially large local kinetic term translates into a small local coupling, but it does not change the location of poles and thresholds associated with the UV degrees of freedom).

In summary, our theoretical bound Eq. (14) leads to a tension between direct limits on the graviton mass and fifthforce experiments. This either rules out $\Lambda_{3}$ massive gravity or implies that the theory is unable to make predictions at scales where GR instead agrees with experimental observations (this EFT of massive gravity cannot tell, e.g., whether an apple would fall to the ground or else go up). This calls for new ideas on extending the theory in the UV, corresponding here to macroscopic distances, a few $10^{5}-10^{6} \mathrm{~km}$ as shown in Fig. 1 , in such a way as to describe the relevant gravitational phenomena while remaining consistent with experimental tests (i.e., the new gravitational dynamics remaining undetected) not only in lunar experiments but also down to the millimeter.

Needless to say, our bounds apply neither to Lorentzviolating models of massive gravity (e.g., Ref. [58]), nor to theories with a massless graviton: one can avoid our bounds by dropping any of the assumptions on the $S$ matrix that led to them. This is in practice not very different from finding explicit UV completions, since it also requires nontrivial dynamics in the UV.

There are several directions where our bounds can find fruitful applications. Immediate ideas involve theories with Goldstone particles, e.g., the EFT for the Goldstino from SUSY breaking or the $R$ axion from $R$ symmetry breaking, and the dilaton from scale symmetry breaking (all of which have interesting phenomenological applications $[29-31,59,60])$, as well as theories with suppressed 2-to-2 amplitudes but unsuppressed 2-to-3 amplitudes, e.g., Ref. [61]. It is also attractive to recast our bounds in diverse spacetime dimensions, such as massive gravity in $d=3[62,63]$, or the conjectured $a$ theorem in $d=6$ (see, e.g., Ref. [64]); for the latter, we have obtained promising preliminary results. Another stimulating avenue is to use our bounds to extend the no-go theorems for massless higher-spin particles in flat space (see, e.g., Refs. [65-69]) to the case of small but finite masses. This could bring new insight on why light higher-spin particles cannot emerge, even in principle, in nongravitational theories without decoupling them or sending the cutoff to zero. One important open question, that for the time being remains elusive, is whether it is possible to extend our results to theories with massless particles with spin $J \geq 2$, thus providing new insights into the long-distance universal properties of the UV completion of quantum gravity, such as string theory, or into IR modifications of GR where the graviton remains massless, such as Horndeski-like theories.

We thank Matt Lewandowski, Matthew McCullough, David Pirtskhalava, Riccardo Rattazzi, Andrew Tolley, Enrico Trincherini, and Filippo Vernizzi for useful discussions. We thank Duccio Pappadopulo, Massimo Porrati, and Gabriele Trevisan for useful comments. We thank Claudia de Rham, Scott Melville, Andrew Tolley, and Shuangyong Zhou for correspondence concerning the Vainshtein mechanism. B. B. thanks Cliff Cheung and Grant Remmen for correspondence. B. B. is supported in part by MIUR-FIRB Grant No. RBFR12H1MW "A new strong force, the origin of masses and the LHC." B. B. thanks Marco Cirelli and the LPTHE for the kind hospitality during the completion of this work, and Roberto Contino and Enrico Trincherini for the kind hospitality at the SNS. J.S. and B. B. would like to express special thanks to the Mainz Institute for Theoretical Physics (MITP) for its hospitality and support.

[1] R. J. Eden, P. V. Landshoff, D. I. Olive, and J. C. Polkinghorne, The Analytic S-matrix (Cambridge University Press, Cambridge, England, 1966).

[2] A. Martin, Rigorous results from theory and unitarity, CERN Report No. CERN-TH-1181, 1970, https://cds .cern.ch/record/944356.

[3] M. Gell-Mann, M. L. Goldberger, and W. E. Thirring, Use of causality conditions in quantum theory, Phys. Rev. 95, 1612 (1954).

[4] M. L. Goldberger, Causality conditions and dispersion relations: 1. Boson fields, Phys. Rev. 99, 979 (1955).

[5] A. Adams, N. Arkani-Hamed, S. Dubovsky, A. Nicolis, and R. Rattazzi, Causality, analyticity and an IR obstruction to UV completion, J. High Energy Phys. 10 (2006) 014.

[6] Z. Komargodski and A. Schwimmer, On renormalization group flows in four dimensions, J. High Energy Phys. 12 (2011) 099.

[7] M. A. Luty, J. Polchinski, and R. Rattazzi, The $a$-theorem and the asymptotics of 4D quantum field theory, J. High Energy Phys. 01 (2013) 152.

[8] A. V. Manohar and V. Mateu, Dispersion relation bounds for $\pi \pi$ scattering, Phys. Rev. D 77, 094019 (2008).

[9] J. Distler, B. Grinstein, R. A. Porto, and I. Z. Rothstein, Falsifying Models of New Physics via $W W$ Scattering, Phys. Rev. Lett. 98, 041601 (2007).

[10] L. Vecchi, Causal versus analytic constraints on anomalous quartic gauge couplings, J. High Energy Phys. 11 (2007) 054.

[11] B. Bellazzini, L. Martucci, and R. Torre, Symmetries, sum rules and constraints on effective field theories, J. High Energy Phys. 09 (2014) 100.

[12] I. Low, R. Rattazzi, and A. Vichi, Theoretical constraints on the Higgs effective couplings, J. High Energy Phys. 04 (2010) 126.

[13] A. Falkowski, S. Rychkov, and A. Urbano, What if the Higgs couplings to $W$ and $Z$ bosons are larger than in the Standard Model? J. High Energy Phys. 04 (2012) 073.

[14] A. Urbano, Remarks on analyticity and unitarity in the presence of a strongly interacting light Higgs, J. High Energy Phys. 06 (2014) 060.

[15] B. Bellazzini, C. Cheung, and G. N. Remmen, Quantum gravity constraints from unitarity and analyticity, Phys. Rev. D 93, 064076 (2016). 
[16] C. Cheung and G. N. Remmen, Positive signs in massive gravity, J. High Energy Phys. 04 (2016) 002.

[17] J. Bonifacio, K. Hinterbichler, and R. A. Rosen, Positivity constraints for pseudolinear massive spin-2 and vector Galileons, Phys. Rev. D 94, 104001 (2016).

[18] B. Bellazzini, Softness, and amplitudes positivity for spinning particles, J. High Energy Phys. 02 (2017) 034.

[19] A. Nicolis, R. Rattazzi, and E. Trincherini, Energy's and amplitudes' positivity, J. High Energy Phys. 05 (2010) 095; Erratum, 11 (2011) 128.

[20] L. Keltner and A. J. Tolley, UV properties of Galileons: Spectral densities, arXiv:1502.05706.

[21] C. de Rham, S. Melville, A. J. Tolley, and S. Y. Zhou, Massive Galileon positivity bounds, J. High Energy Phys. 09 (2017) 072.

[22] D. Baumann, D. Green, H. Lee, and R. A. Porto, Signs of analyticity in single-field inflation, Phys. Rev. D 93, 023523 (2016).

[23] D. Croon, V. Sanz, and J. Setford, Goldstone inflation, J. High Energy Phys. 10 (2015) 020.

[24] C. Cheung and G. N. Remmen, Infrared consistency and the weak gravity conjecture, J. High Energy Phys. 12 (2014) 087.

[25] C. Cheung and G. N. Remmen, Naturalness and the Weak Gravity Conjecture, Phys. Rev. Lett. 113, 051601 (2014).

[26] Z. Komargodski, M. Kulaxizi, A. Parnachev, and A. Zhiboedov, Conformal field theories and deep inelastic scattering, Phys. Rev. D 95, 065011 (2017).

[27] T. Hartman, S. Jain, and S. Kundu, Causality constraints in conformal field theory, J. High Energy Phys. 05 (2016) 099.

[28] L. F. Alday and A. Bissi, Unitarity and positivity constraints for CFT at large central charge, J. High Energy Phys. 07 (2017) 044.

[29] S. Bruggisser, F. Riva, and A. Urbano, Strongly interacting light dark matter, SciPost Phys. 3, 017 (2017).

[30] B. Bellazzini, A. Mariotti, D. Redigolo, F. Sala, and J. Serra, $R$-Axion at Colliders, Phys. Rev. Lett. 119, 141804 (2017).

[31] B. Bellazzini, F. Riva, J. Serra, and F. Sgarlata, The other fermion compositeness, J. High Energy Phys. 11 (2017) 020.

[32] C. de Rham, S. Melville, A. J. Tolley, and S. Y. Zhou, Positivity bounds for scalar theories, Phys. Rev. D 96, 081702 (2017).

[33] C. de Rham, S. Melville, A. J. Tolley, and S. Y. Zhou, UV complete me: Positivity bounds for particles with spin, J. High Energy Phys. 03 (2018) 011.

[34] A. Nicolis, R. Rattazzi, and E. Trincherini, The Galileon as a local modification of gravity, Phys. Rev. D 79, 064036 (2009).

[35] D. Pirtskhalava, L. Santoni, E. Trincherini, and F. Vernizzi, Weakly broken Galileon symmetry, J. Cosmol. Astropart. Phys. 09 (2015) 007.

[36] C. de Rham and G. Gabadadze, Generalization of the FierzPauli action, Phys. Rev. D 82, 044020 (2010).

[37] C. de Rham, G. Gabadadze, and A. J. Tolley, Resummation of Massive Gravity, Phys. Rev. Lett. 106, 231101 (2011).

[38] M. Froissart, Asymptotic behavior and subtractions in the Mandelstam representation, Phys. Rev. 123, 1053 (1961).

[39] A. Martin, Extension of the axiomatic analyticity domain of scattering amplitudes by unitarity: 1, Nuovo Cimento A 42, 930 (1966).
[40] K. Hinterbichler, Theoretical aspects of massive gravity, Rev. Mod. Phys. 84, 671 (2012).

[41] C. de Rham, Massive gravity, Living Rev. Relativity 17, 7 (2014).

[42] S. F. Hassan and R. A. Rosen, Resolving the Ghost Problem in Non-Linear Massive Gravity, Phys. Rev. Lett. 108, 041101 (2012).

[43] N. Arkani-Hamed, H. Georgi, and M. D. Schwartz, Effective field theory for massive gravitons and gravity in theory space, Ann. Phys. (Amsterdam) 305, 96 (2003).

[44] See Supplemental Material at http://link.aps.org/ supplemental/10.1103/PhysRevLett.120.161101 for more details on the scattering amplitudes and the bounds, which includes Refs. [45-51].

[45] X. O. Camanho, J. D. Edelstein, J. Maldacena, and A. Zhiboedov, Causality constraints on corrections to the graviton three-point coupling, J. High Energy Phys. 02 (2016) 020.

[46] C. Cheung and G. N. Remmen, Positivity of CurvatureSquared Corrections in Gravity, Phys. Rev. Lett. 118, 051601 (2017).

[47] K. Benakli, S. Chapman, L. Darme, and Y. Oz, Superluminal graviton propagation, Phys. Rev. D 94, 084026 (2016).

[48] X. O. Camanho, G. Lucena Gómez, and R. Rahman, Causality constraints on massive gravity, Phys. Rev. D 96, 084007 (2017).

[49] K. Hinterbichler, A. Joyce, and R. A. Rosen, Massive spin-2 scattering and asymptotic superluminality, J. High Energy Phys. 03 (2018) 051.

[50] G. F. Giudice, C. Grojean, A. Pomarol, and R. Rattazzi, The strongly interacting light Higgs, J. High Energy Phys. 06 (2007) 045.

[51] A. G. Cohen, D. B. Kaplan, and A. E. Nelson, Counting $4 \pi$ 's in strongly coupled supersymmetry, Phys. Lett. B 412, 301 (1997).

[52] C. de Rham, J. T. Deskins, A. J. Tolley, and S. Y. Zhou, Graviton mass bounds, Rev. Mod. Phys. 89, 025004 (2017).

[53] G. Dvali, A. Gruzinov, and M. Zaldarriaga, The accelerated universe and the moon, Phys. Rev. D 68, 024012 (2003).

[54] J. G. Williams, S. G. Turyshev, and D. H. Boggs, Progress in Lunar Laser Ranging Tests of Relativistic Gravity, Phys. Rev. Lett. 93, 261101 (2004).

[55] A. I. Vainshtein, To the problem of nonvanishing gravitation mass, Phys. Lett. B 39B, 393 (1972).

[56] C. Deffayet, G. R. Dvali, G. Gabadadze, and A. I. Vainshtein, Nonperturbative continuity in graviton mass versus perturbative discontinuity, Phys. Rev. D 65, 044026 (2002).

[57] A. Nicolis and R. Rattazzi, Classical and quantum consistency of the DGP model, J. High Energy Phys. 06 (2004) 059.

[58] D. Blas and S. Sibiryakov, Completing Lorentz violating massive gravity at high energies, Zh. Eksp. Teor. Fiz. 147, 578 (2015) [J. Exp. Theor. Phys. 120, 509 (2015)].

[59] B. Bellazzini, C. Csaki, J. Hubisz, J. Serra, and J. Terning, A naturally light dilaton and a small cosmological constant, Eur. Phys. J. C 74, 2790 (2014).

[60] B. Bellazzini, C. Csaki, J. Hubisz, J. Serra, and J. Terning, A Higgs-like dilaton, Eur. Phys. J. C 73, 2333 (2013). 
[61] C. Cheung, K. Kampf, J. Novotny, C. H. Shen, and J. Trnka, A periodic table of effective field theories, J. High Energy Phys. 02 (2017) 020.

[62] E. A. Bergshoeff, O. Hohm, and P. K. Townsend, Massive Gravity in Three Dimensions, Phys. Rev. Lett. 102, 201301 (2009).

[63] S. Deser, R. Jackiw, and S. Templeton, Topologically massive gauge theories, Ann. Phys. (N.Y.) 140, 372 (1982); 281, 409 (2000); Erratum, 185, 406 (1988).

[64] H. Elvang, D. Z. Freedman, L. Y. Hung, M. Kiermaier, R. C. Myers, and S. Theisen, On renormalization group flows and the $a$-theorem in 6D, J. High Energy Phys. 10 (2012) 011.
[65] S. Weinberg, The Quantum Theory of Fields, Vol. 1: Foundations (Cambridge University Press, Cambridge, England, 2005).

[66] S. Weinberg, Photons and gravitons in $S$-matrix theory: Derivation of charge conservation and equality of gravitational and inertial mass, Phys. Rev. 135, B1049 (1964).

[67] M. T. Grisaru, H. N. Pendleton, and P. van Nieuwenhuizen, Supergravity and the $S$-matrix, Phys. Rev. D 15, 996 (1977).

[68] M. Porrati, Universal limits on massless high-spin particles, Phys. Rev. D 78, 065016 (2008).

[69] M. Porrati, Old and new no-go theorems on interacting massless particles in flat space, arXiv:1209.4876. 